\title{
Manuscript
}

\section{Catalytic asymmetric reductive alkylation of enamines to chiral aliphatic amines}

Jia-Wang Wang, ${ }^{\dagger}$ Yan Li, ${ }^{\dagger}$ Wan Nie, Zhe Chang, Zi-An Yu, Yi-Fan Zhao, Xi Lu, ${ }^{*}$ Yao Fu*

Hefei National Laboratory for Physical Sciences at the Microscale, CAS Key Laboratory of Urban Pollutant Conversion, Anhui Province Key Laboratory of Biomass Clean Energy, iChEM, University of Science and Technology of China, Hefei 230026, China

*E-mail: luxi@mail.ustc.edu.cn; fuyao@ustc.edu.cn.

†J.-W.W. and Y.Li contributed equally.

To increase the reliability and success rate of drug discovery, efforts have been made to increase the $\mathrm{C}\left(s p^{3}\right)$ fraction and avoid flat molecules. $s p^{3}$-rich enantiopure amines are most frequently encountered as chiral auxiliaries, synthetic intermediates for pharmaceutical agents and bioactive natural products. Streamlined construction of chiral aliphatic amines has long been regarded as a paramount challenge. Mainstream approaches, including hydrogenation of enamines and imines, $\mathrm{C}-\mathrm{H}$ amination, and alkylation of imines, were applied for the synthesis of chiral amines with circumscribed skeleton structures; typically, the chiral carbon centre was adjacent to an auxiliary aryl or ester group. Herein, we report a mild and general nickel-catalysed asymmetric reductive alkylation to effectively convert enamines-a class of important yet underexploited feedstock chemicals-into drug-like $\alpha$-branched chiral amines and derivatives. This reaction involves the regio- and stereoselective hydrometallation of an enamine to generate a catalytic amount of enantioenriched alkylnickel intermediate, followed by $\mathrm{C}-\mathrm{C}$ bond formation via alkyl electrophiles.

Enantiopure amines are frequently encountered as chiral auxiliaries and synthetic intermediates for pharmaceutical agents and bioactive natural products ${ }^{1,2}$. Nearly half of small- 
molecule pharmaceuticals among the top 200 drugs by retail sales in 2019 contain an enantioenriched aliphatic amine as the key structural element ${ }^{3}$. (Fig. 1a) To synthesize optically pure amines efficiently, exciting strides have been made in the field of hydrogenation of enamines and imines ${ }^{4-7}, \mathrm{C}-\mathrm{H}$ amination ${ }^{8-10}$, and hydroamination of alkenes ${ }^{11-14}$ to supplement the nucleophilic substitution reactions of the corresponding chiral alcohols. However, the primary problem, inadequate substrate scope, still remains. It is quite difficult to distinguish two substituents with minimal differences in their steric and electronic properties with simple catalysts to achieve good enantioselectivity ${ }^{15-19}$. Thus, these methods were applied for the synthesis of chiral amines with circumscribed skeleton structures; typically, the chiral carbon centre was adjacent to an auxiliary $\operatorname{ary}^{19}$ or ester group ${ }^{20}$. Another prevalent method to selectively synthesize enantioenriched amides is the catalytic asymmetric alkylation of imines through carbon-carbon bond formation. However, only a few catalytic enantioselective alkylations of imines with particular hyperactive alkylation reagents, typically relatively air- and moisture-sensitive Grignard reagents ${ }^{21}$ or zinc reagents ${ }^{22,23}$, have been reported. However, poorly electrophilic alkyl-substituted imines presented poor reactivity, and the C-N double bond geometrical isomer mixture led to unsatisfactory enantioselectivities. (Fig. 1b)

In designing a synthetically useful method for synthesizing $s p^{3}$-rich chiral amines and derivatives to meet the increased demand in drug discovery, we innovated an operationally facile and fundamentally unique reaction using readily available and batch-stable enamines and alkyl halides under reductive conditions. Since 2016, our group devoted efforts to the development of olefin reductive alkylation reactions ${ }^{24-28}$, and olefin reductive alkylation has been one of the most efficient and prevailing methods for alkyl-alkyl formation ${ }^{29-32}$. Herein we report a significant breakthrough to reductive hydroalkylation of generalized organic unsaturated systems, namely, the enamine reductive hydroalkylation process involves in situ hydrometallation of enamine to generate prospective enantioenriched alkylnickel intermediates, which subsequently react with alkyl halides to form $\mathrm{C}\left(s p^{3}\right)-\mathrm{C}\left(s p^{3}\right)$ bonds. This reaction enables the construction of structurally complex and multifunctional amides and $\beta$ aminoboronates with excellent functional-group compatibility suitable for late-stage elaboration applications. This reaction also facilitates the design and development of drug-like nitrogen-containing chiral molecules that are difficult to access by other synthetic routes, resulting in widespread application in the pharmaceutical sector and synthetic chemistry field. (Fig. 1c) 


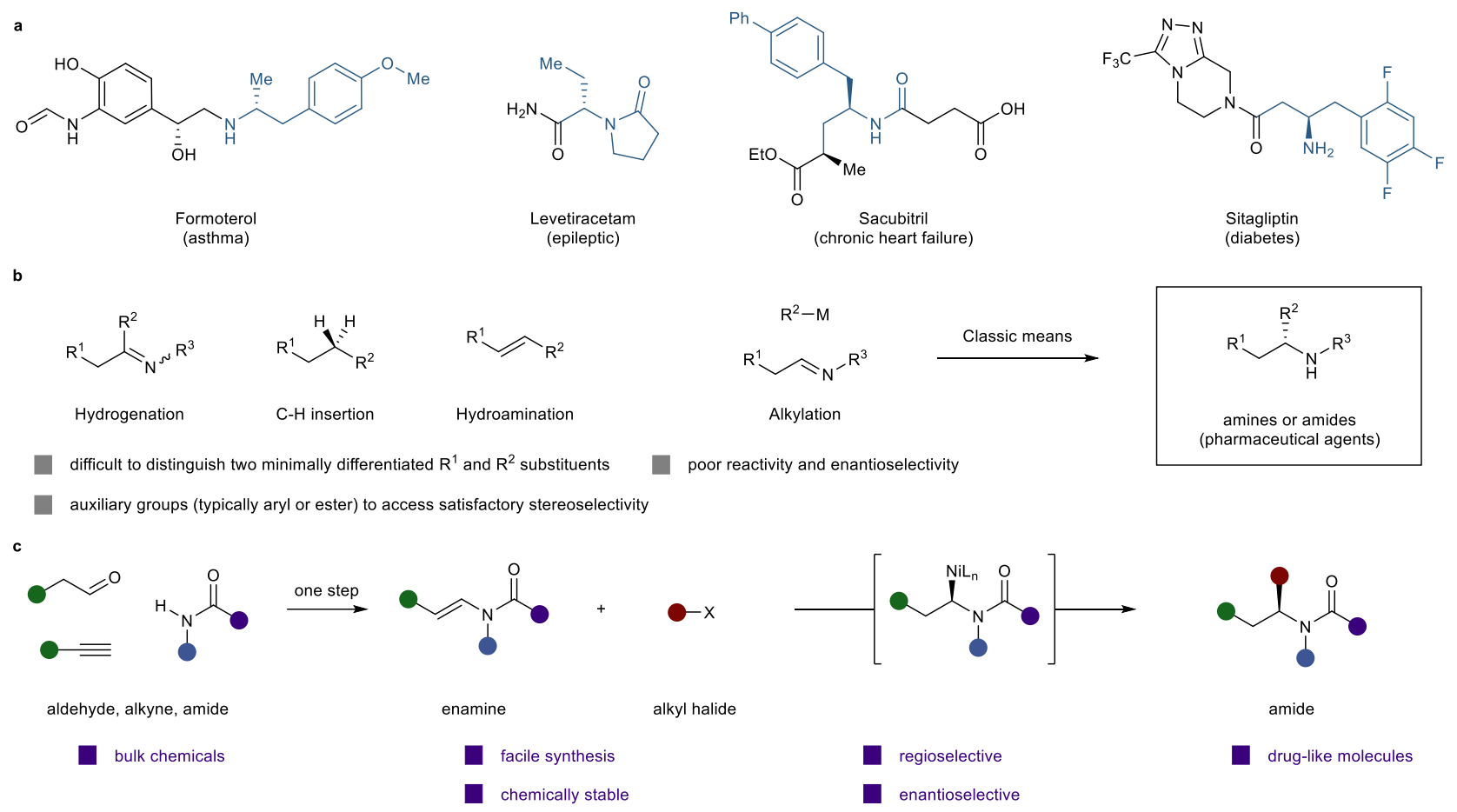

Fig. 1 Design of a modular enamine reductive alkylation for the synthesis of chiral aliphatic amines. a, Representative drug molecules demonstrating the universal existence of chiral aliphatic amines in biologically active molecules. b. Catalytic asymmetric reductive alkylation of readily available and chemically stable enamines enables rapid access to the privileged chiral aliphatic amines, complementing mainstream approaches that are limited by auxiliary groups to access satisfactory stereoselectivity. c, Synthetic analysis and proposed mechanism of enamine reductive alkylation. Enamines are prepared via facile syntheses from bulk chemicals-aldehydes, alkynes, and amides - and are batch stable in laboratory environments. It was assumed that this reaction involved regio- and stereoselective hydrometallation of an enamine to generate a catalytic amount of enantioenriched alkylnickel intermediate, followed by $\mathrm{C}-\mathrm{C}$ bond formation with alkyl electrophiles to yield chiral amines.

At the beginning of this study, we determined that this enamine reductive alkylation could occur when a chiral nickel/bisoxazoline catalyst was combined with diethoxymethylsilane (DEMS) and $\mathrm{KF}$ in a DMAc/tBuOH mixed solvent. Under our optimized conditions, a fairly broad scope of $N$-alkyl- $N$-acyl enamines served as active substrates to deliver the desired products. (Fig. 2) Although the amino-protecting groups varied in terms of steric and electronic properties, high yields (70-94\% yield) and high enantioselectivities [86-97\% ee (enantiomeric excess)] were achieved in all cases (1-12). The alkyl substituents on the enamine were also investigated; steric hindrance has a typical effect on the coupling efficiency (13-17). In the case of less bulky alkyl substituents, good yields were achieved, and vice versa. The enantioselectivity was hardly affected by the alkyl substituents, with the only exception being dialkylsubstituted enamines (18), which were produced in moderate yield with complete loss of enantioselectivity. $N$-Styrylenamines (16-17), which exhibit competitive reactivity at the benzylic position, were also good substrates to deliver the single $N$ - $\alpha$-alkylation selective products.

With respect to alkyl electrophiles, both alkyl iodides and alkyl bromides could be 
converted efficiently (19-33). Relatively inert alkyl bromides $(\mathbf{1 9}, \mathbf{2 1 - 2 2})$ required higher reaction temperatures and a NaI additive to achieve satisfactory outcomes. A wide array of functional groups were compatible; for example, a phthalimide (22), an alkyl ester moiety (23), an amide possessing $\mathrm{N}-\mathrm{H}$ bonds (24-25), and a base-sensitive ketone (26) were well tolerated. This reaction could also be conducted in the presence of an aryl chloride (27), an alkyl chloride (28), and an aryl bromide (29), thus providing an exceptional opportunity for further transformation at the preserved carbon-halogen bonds. In addition, several highly reactive electron-rich heterocycles (30-32) - which could undergo facile Friedel-Crafts reaction or C-H activationwere retained during the transformation. Finally, the late-stage diversification of complex molecules illustrated the high degree of compatibility of diverse functional groups for this newly developed enamine reductive alkylation (34-37). 


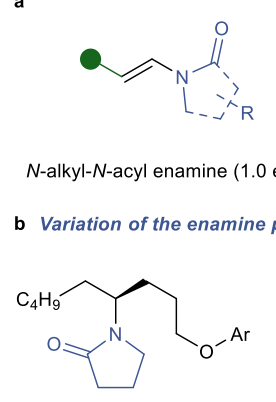

7, $70 \%$ yield, $93 \% \mathrm{ee}^{a}$
$1,93 \%$ yield, $96 \% \mathrm{ee}^{a}$

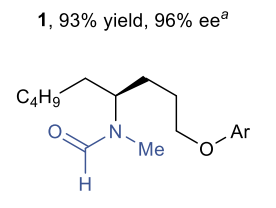

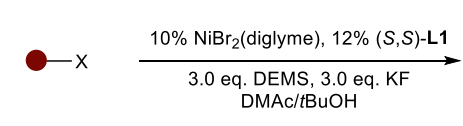

alkyl halide (2.0 eq.)

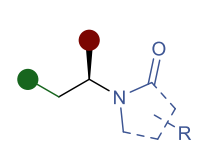

amide
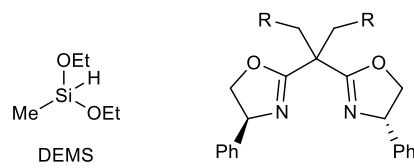

$(S, S)-\mathbf{L} 1, \mathrm{R}=4-\mathrm{tBuC}_{6} \mathrm{H}_{4}$

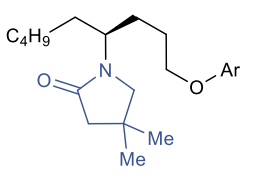

$2,86 \%$ yield, $97 \%$ ee $^{a}$

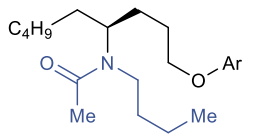

8, $94 \%$ yield, $90 \%$ ee $^{a}$

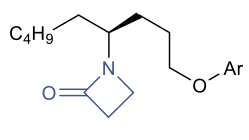

3, $91 \%$ yield, $89 \%$ ee

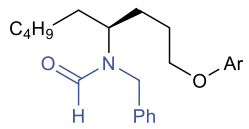

9, $70 \%$ yield, $91 \%$ ee $^{a}$

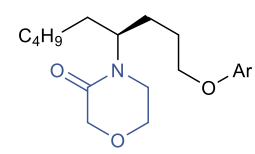

4, $90 \%$ yield, $98 \%$ ee $^{b}$

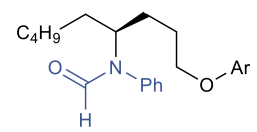

10, $90 \%$ yield, $91 \% \mathrm{ee}^{a}$

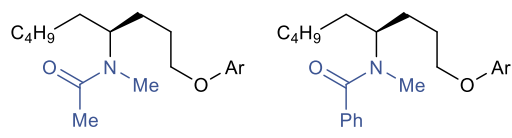

$5,90 \%$ yield, $92 \%$ ee $^{a}$

6, $90 \%$ yield, $92 \% \mathrm{ee}^{a}$

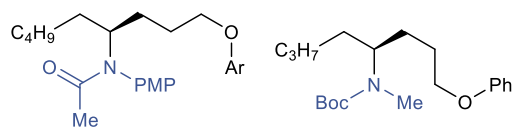

$11,82 \%$ yield, $94 \%$ ee $\quad 12,75 \%$ yield, $86 \% \mathrm{ee}^{b}$

Variation of the enamine aliphatic substituents

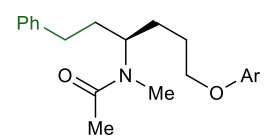

${ }^{\mathrm{N}}>$

$14,70 \%$ yield, $91 \% \mathrm{ee}^{\mathrm{a}}$

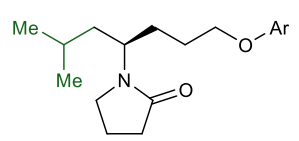

$15,40 \%$ yield, $93 \% \mathrm{ee}^{\mathrm{a}}$

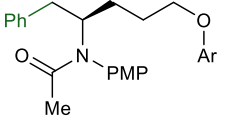

$16,60 \%$ yield, $97 \% \mathrm{ee}^{a}$

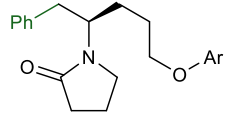

$17,91 \%$ yield, $97 \% \mathrm{ee}^{\mathrm{a}}$

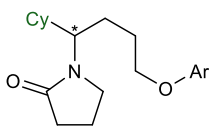

$18,54 \%$ yield, $<2 \%$ ee ${ }^{a}$

Variation of the alkyl halides<smiles>CC(=O)N(C)[C@@H](CCCCN)CCc1ccccc1</smiles>

19, $(\mathrm{X}=\mathrm{Br}) 82 \%$ yield, $89 \% \mathrm{ee}^{\mathrm{c}}$

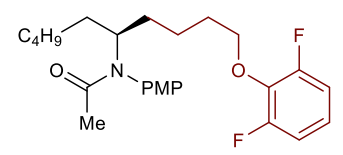

20, $72 \%$ yield, $91 \% \mathrm{ee}^{\mathrm{a}}$

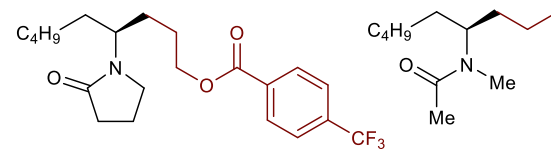

22, $(\mathrm{X}=\mathrm{Br}) 60 \%$ yield, $90 \% \mathrm{ee}^{\mathrm{c}}$
21, $(\mathrm{X}=\mathrm{Br}) 95 \%$ yield, $95 \% \mathrm{ee}^{\mathrm{c}}$

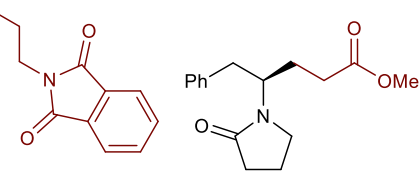

23, $70 \%$ yield, $96 \%$ ee $^{a}$<smiles>CCCCCCC(CCCCCC)N(C(C)=O)C(C)=O</smiles>

24, $85 \%$ yield, $96 \%$ ee

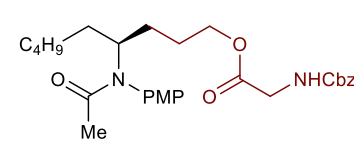

$25,90 \%$ yield, $95 \%$ ee

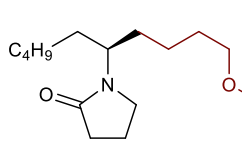

26, $50 \%$ yield, $96 \%$ ee

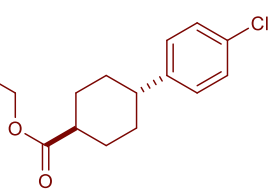

$27,92 \%$ yield, $96 \%$ ee $^{a}$

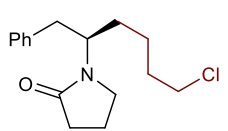

$28,92 \%$ yield, $98 \%$ ee $^{a}$

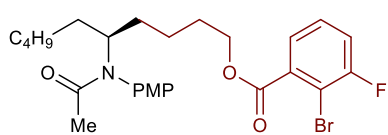

29, $90 \%$ yield, $96 \%$ ee $^{a}$

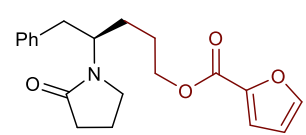

30, $(\mathrm{X}=\mathrm{Br}) 82 \%$ yield, $97 \%$ ee $^{\mathrm{c}}$

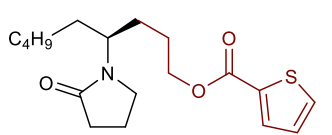

31, $(\mathrm{X}=\mathrm{Br}) 91 \%$ yield, $97 \% \mathrm{ee}^{\mathrm{c}}$

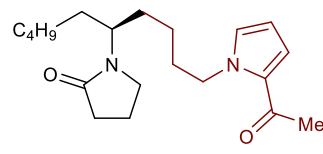

32, $70 \%$ yield, $97 \%$ ee $\mathrm{e}^{a}$

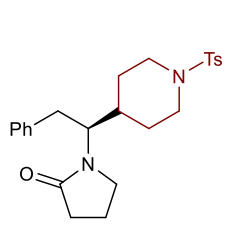

33, $70 \%$ yield, $87 \%$ ee $^{d}$

Modification of complex moleculas
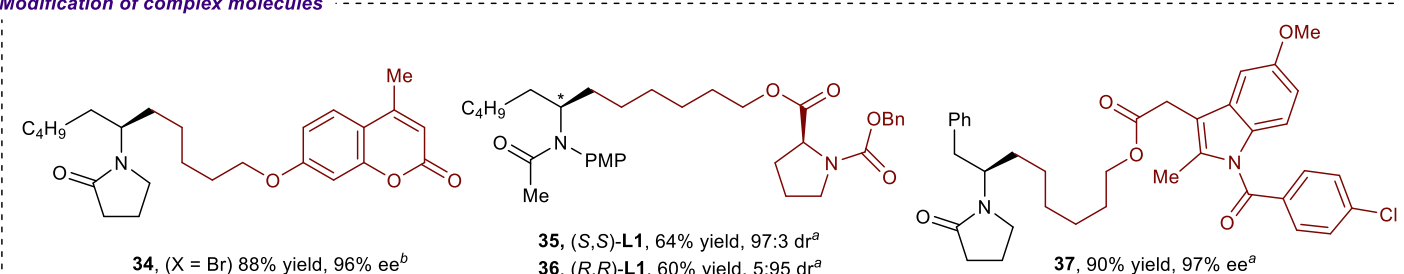

$37,90 \%$ yield, $97 \%$ ee

Fig. 2 Asymmetric reductive alkylation of $N$-alkyl- $N$-acyl enamines. a, Optimized conditions for the reductive alkylation of $N$-alkyl- $N$-acyl enamines. b, Scope of the reductive alkylation of $N$-alkyl- $N$-acyl enamines. ${ }^{a} \mathrm{DMAc}: t \mathrm{BuOH}(5: 4,0.25 \mathrm{M}), 25{ }^{\circ} \mathrm{C}, 20$ hours, isolated yield, o.20 mmol scale; ${ }^{b} \mathrm{DMAc}: t \mathrm{BuOH}(5: 4,0.25 \mathrm{M}), 5{ }^{\circ} \mathrm{C}$, 30 hours, isolated yield, $0.20 \mathrm{mmol}$ scale; ${ }^{c} \mathrm{DMAc}: \mathrm{tBuOH}(5: 4,0.25 \mathrm{M})$, NaI (o.5 eq.), $35^{\circ} \mathrm{C}, 20$ hours, isolated yield, $0.20 \mathrm{mmol}$ scale; ${ }^{d} \mathrm{DMAc}: \mathrm{BuOH}(5: 4,0.25 \mathrm{M}), 50{ }^{\circ} \mathrm{C}$, 20 hours, isolated yield, $0.20 \mathrm{mmol}$ scale. Ar = 2-naphthyl. Diglyme $=2$-methoxyethyl ether. DMAc $=N, N$-dimethylacetamide. $\mathrm{PMP}=p$-methoxyphenyl. Boc $=$ tertbutoxycarbonyl. $\mathrm{Cbz}=$ carbobenzyloxy. $\mathrm{Ts}=$ tosyl. $\mathrm{Bn}=$ benzyl. $\mathrm{dr}=$ diastereomeric ratio.

Next, we aimed to expand the scope of asymmetric reductive alkylation to include a second family of enamines, specifically, $\mathrm{N}-\mathrm{H}-\mathrm{N}$-acyl enamines possessing $\mathrm{N}-\mathrm{H}$ bonds (Fig. 3). Whereas 
reactions using the aforementioned method result in poor yield and enantioselectivity, as depicted in Fig. 2, $\mathrm{N}$-H-N-acyl enamines performed well under modified conditions after considerable effort. The scope of $\mathrm{N}-\mathrm{H}-\mathrm{N}$-acyl enamine reductive alkylation proved extremely broad with respect to both the enamine and the alkyl halide (38-67). In the case of $\mathrm{N}-\mathrm{H}-\mathrm{N}$-acyl enamine, the amino-protecting group and aliphatic substituent could be altered and could tolerate many functional groups without any detrimental effect on the coupling yield or enantioselectivity (38-55). The improved flexibility of the amino-protecting groups, especially the ability to work with Boc (tert-butoxycarbonyl) protecting groups (e.g., 43-48, 50-53, 58-67), ameliorated the practicability and applicability of this reaction. Furthermore, even more sterically encumbered dialkylsubstituted $N$-H-N-acyl enamines $(\mathbf{4 7}-51)$ could be converted to the desired products with excellent enantioselectivities, which was significantly different from the results obtained when using $N$-alkyl- $N$-acyl enamines. Similarly, a wide range of synthetically useful functional groups, such as an internal alkene (52-53), an aryl fluoride (57), a trifluoromethyl (58), and a cyano (6o), were tolerated. Privileged heterocyclic motifs such as thiophene (64), furan (65), pyrrole (66), and coumarin (67) are commonly found in medicinal drugs and pose no problems during transformation. 


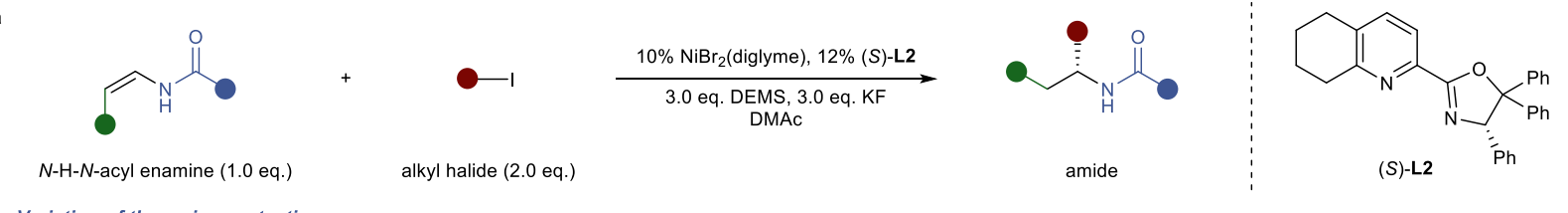

b Variation of the amino protecting groups

$\overbrace{\mathrm{Me}}^{\mathrm{O}}$

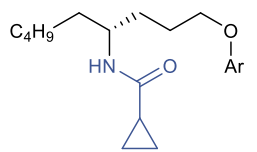

$39,80 \%$ yield, $93 \%$ ee $^{a}$

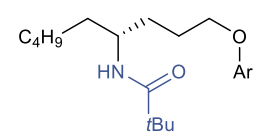

$40,91 \%$ yield, $86 \%$ ee $^{a}$

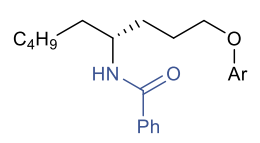

$41,57 \%$ yield, $86 \%$ ee $^{a}$

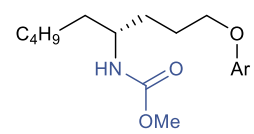

42, $62 \%$ yield, $91 \%$ ee

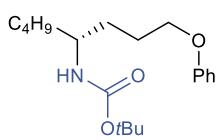

$43,88 \%$ yield, $92 \% \mathrm{ee}^{a}$

Variation of the enamine aliphatic substituents

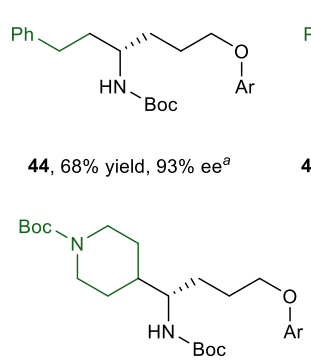

$50,52 \%$ yield, $92 \%$ ee $63 \%$ yield, $89 \%$ ee $^{b}$

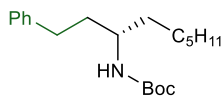

$45,66 \%$ yield, $93 \% \mathrm{ee}^{\mathrm{a}}$

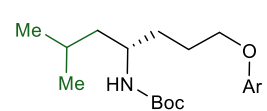

46, $78 \%$ yield, $93 \% \mathrm{ee}^{\mathrm{a}}$

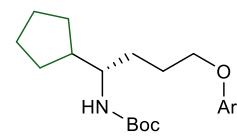

$47,65 \%$ yield, $97 \%$ ee $^{a}$

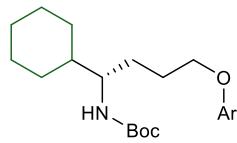

48, $42 \%$ yield, $97 \%$ ee $^{a}$ $63 \%$ yield, $95 \%$ ee $^{b}$

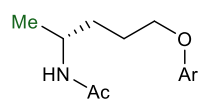

$\mathbf{5 4}, 82 \%$ yield, $85 \% \mathrm{ee}^{\mathrm{a}}$

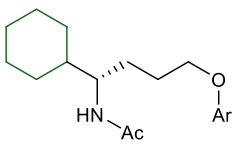

49, $50 \%$ yield, $86 \%$ ee $^{b}$

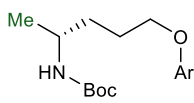

$\mathbf{5 5}, 69 \%$ yield, $88 \%$ ee ${ }^{a}$ Variation of the alkyl halides<smiles>O=C(N[C@H](CCCCOC(=O)OCc1ccccc1)Cc1ccccc1)OCc1ccccc1</smiles>

56, $60 \%$ yield, $91 \%$ ee

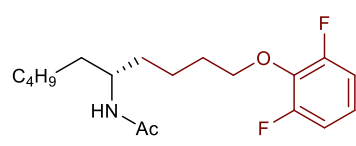

57, $78 \%$ yield, $90 \% \mathrm{ee}^{a}$

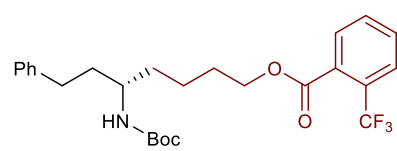

$\mathbf{5 8}, 66 \%$ yield, $93 \%$ ee

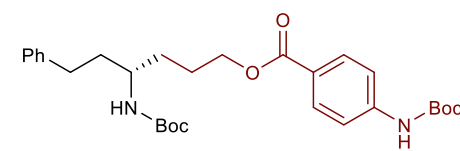

59, $60 \%$ yield, $92 \% \mathrm{ee}^{a}$

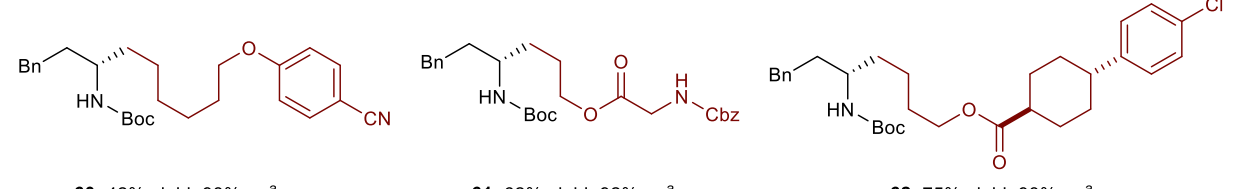

60, $42 \%$ yield, $93 \%$ ee

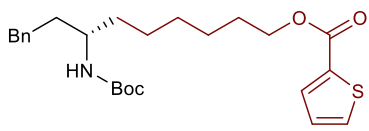

64, 59\% yield, $91 \%$ ee $^{a}$

$$
\text { 61, } 62 \% \text { yield, } 92 \% \mathrm{ee}^{a}
$$

$65,62 \%$ yield, $93 \%$ ee $^{a}$
62, $75 \%$ yield, $93 \% \mathrm{ee}^{\mathrm{a}}$

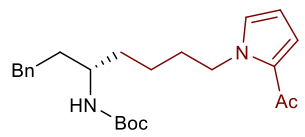

$66,50 \%$ yield, $95 \% \mathrm{ee}^{\mathrm{a}}$

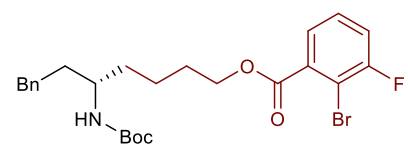

$63,62 \%$ yield, $91 \%$ ee<smiles>Cc1cc(=O)oc2cc(OCCCC[C@H](Cc3ccccc3)NC(=O)c3ccccc3)ccc12</smiles>

$67,68 \%$ yield, $92 \% \mathrm{ee}^{a}$

Fig. 3 Asymmetric reductive alkylation of $\mathrm{N}-\mathrm{H}-\mathrm{N}$-acyl enamines. a, Optimized conditions for the reductive alkylation of $\mathrm{N}-\mathrm{H}-\mathrm{N}$-acyl enamines. b, Scope of the reductive alkylation of $\mathrm{N}-\mathrm{H}-\mathrm{N}$-acyl enamines. ${ }^{a} \mathrm{DMAc}(\mathrm{o.2} \mathrm{M})$, $-27^{\circ} \mathrm{C}$, 40 hours, isolated yield, $0.20 \mathrm{mmol} \mathrm{scale;}{ }^{b} \mathrm{DMAc}(\mathrm{o.2} \mathrm{M}), \mathrm{o}^{\circ} \mathrm{C}, 4 \mathrm{o}$ hours, isolated yield, $0.20 \mathrm{mmol}$ scale. $\mathrm{Ar}=$ 2-naphthyl. Ac = acetyl. Bz = benzoyl.

To examine the reaction mechanism of enamine reductive alkylation, we carried out radical clock experiments. (Fig. 4a) Alkyl iodide (69) containing a cyclopropyl ring is a frequently used radical clock substrate and was applied under standard reaction conditions. The ring-opened product (70) was obtained in $80 \%$ isolated yield, though the hypothetical terminal double bond migrated to the interior. We also tested the reductive alkylation of 5-iodopent-1-ene (72). The assumed ring-cyclized product (74) was not observed, and a complex mixture of linear coupling products (73) was obtained, which revealed that the migration of vinyl double bonds was a very rapid primitive step. Despite all this, it was concluded that the activation of alkyl halides proceeded through a radical pathway. Then, we took advantage of deuterated silane $\left(\mathrm{Ph}_{2} \mathrm{SiD}_{2}\right)$ 
to study the stereochemistry of this reaction. (Fig. 4b) Deuterium-labelling experiments revealed that the enamine reductive alkylation was completely diastereoselective. The stereochemical results indicated that this reaction proceeded through $\mathrm{Ni}-\mathrm{D}$ intermediate formation and syn-addition to an enamine to generate prospective enantioenriched alkylnickel intermediates ${ }^{33}$. Another observation was that the major enantiomer of the product was determined by the configuration of the ligands. (Fig. 4c) In the case of $N$-alkyl-N-acyl enamines (82), the $(E)$ - or $(Z)$-enamines markedly affected the enantioselectivities. The $(E)$-substrate afforded a much higher level of enantioselectivity. Different from the $N$-alkyl- $N$-acyl enamines, in the case of $\mathrm{N}-\mathrm{H}-\mathrm{N}$-acyl enamines $(68),(E)$ - or $(Z)$-enamines alone or an equimolar mixture of both (E)- and (Z)-enamine afforded comparable coupling yields and enantioselectivities. Finally, DFT calculations were carried out, and the simulation results were highly consistent with the results of the mechanistic study. (Fig. 4d) The optimized structures and relative Gibbs free energies of the four transition states in the $\mathrm{Ni}-\mathrm{H}$ insertion step are summarized in Fig. $4 \mathrm{~d}$. As shown, TS- $\alpha 2$ corresponds to the major $(R)-N$ - $\alpha$-alkylation product, and it has the lowest free energy, which is in line with the experimental outcomes. The favoured transition state TS$\boldsymbol{\alpha} \mathbf{2}$ has larger $\mathrm{C}-\mathrm{H}$ bond distances between methyl groups of the substrate and phenyl groups of the ligand (highlighted by green), resulting in lower steric hindrance than all the other three competing transition states (corresponding to $(S)-N$ - $\alpha$-alkylation products or $N$ - $\beta$-alkylation products, respectively). In addition, we also examined the effect of the $(Z)$-configuration on the enantioselectivity by calculating TS- $\alpha \mathbf{1}$ and TS- $\alpha \mathbf{2}$ with the (Z)-substrate (see supplement information for more details). The free energy gap between TS- $\alpha \mathbf{1}-\mathbf{Z}$ and TS- $\alpha \mathbf{2}-\mathbf{Z}$ (Z signifies the (Z)-substrate) is decreased to $1.6 \mathrm{kcal} / \mathrm{mol}$ compared to that of $2.9 \mathrm{kcal} / \mathrm{mol}$ between TS- $\boldsymbol{\alpha} \mathbf{1}$ and TS- $\alpha_{2}$, which is consistent with the higher level of enantioselectivity of the $(E)$-substrate. Together, the mechanistic study and DFT calculations confirmed our proposed mechanism and reaction design, as shown in Fig. 1c. 


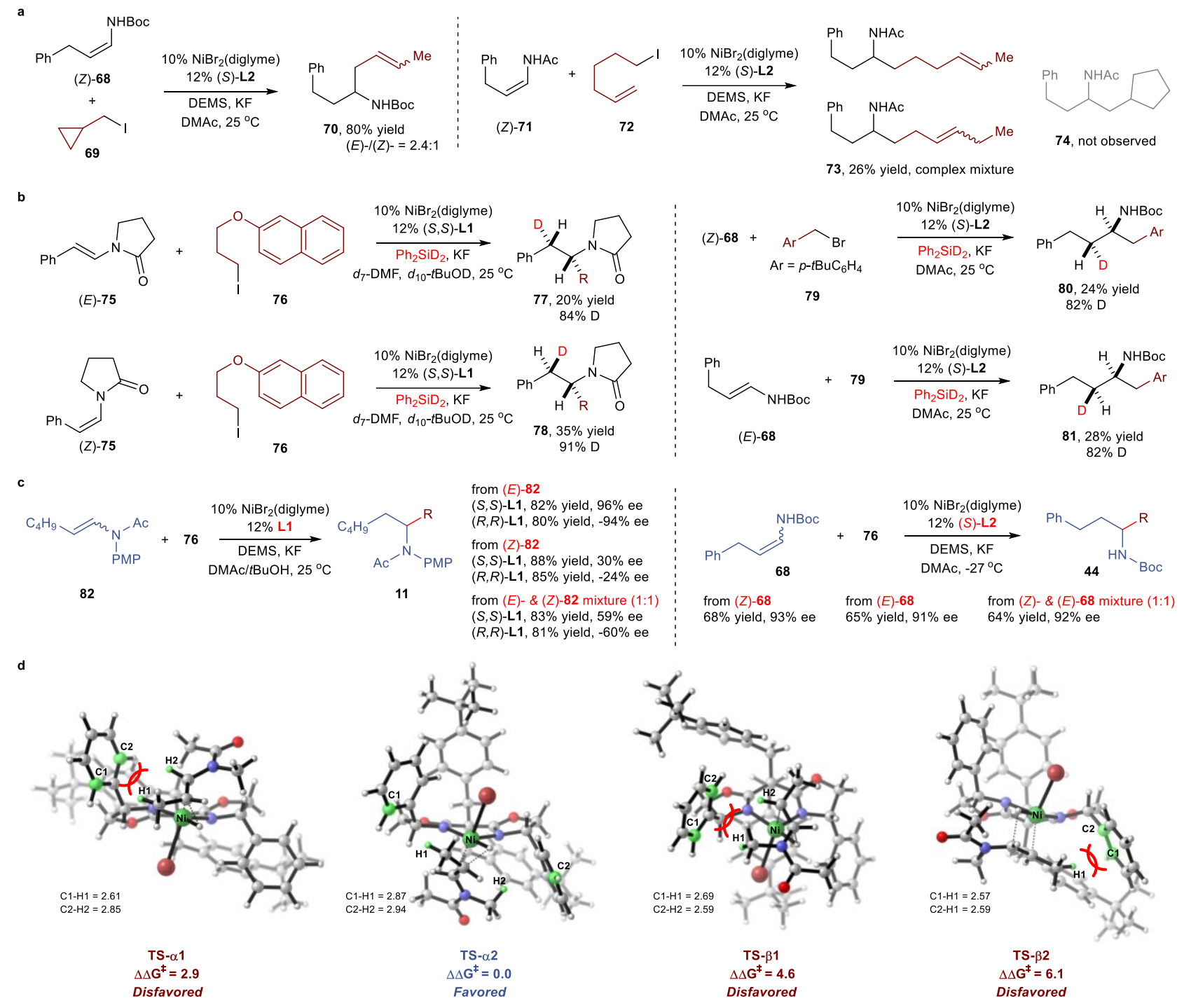

Fig. 4 Mechanistic studies of enamine reductive alkylation. a, Radical clock experiments. b, Deuterium-labelling experiments. c, Configuration effect studies. d, DFT calculations. DFT calculations were also performed at the B $_{3} \mathrm{LYP}_{3} \mathrm{D}_{3}(\mathrm{BJ}) / 6-311 \mathrm{G}(\mathrm{d}, \mathrm{p})-S D D-S M D(D M A) / / \mathrm{B}_{3} \mathrm{LYP}_{2} \mathrm{D}_{3}(\mathrm{BJ}) / 6-31 \mathrm{G}^{*}$-LANL2DZ-SMD(DMA) level of theory. For more details for reaction conditions, see supplement information.

Chiral amino alcohol skeleton structures are ubiquitously found in bioactive natural products or drug molecules. They are also extensively used as chiral pools, ligands or catalysts for asymmetric catalysis. However, the efficient synthesis of enantiopure amino alcohols remains a persistent challenge. For instance, the best known route to obtain amino alcoholsreduction of amino acids-requires prior multistep synthesis to access non-natural amino acids using the highly toxic metal cyanide ${ }^{34}$. Building on our preceding foundation, we establish that enamine reductive alkylation with racemic $\alpha$-haloboronates to access $\beta$-aminoboronates can be accomplished. (Fig. 5, 83-94) Our method, based on classical alkylborate transformations and the Mitsunobu reaction, provided a modular and simplified strategy for the synthesis of chiral amino alcohols and other useful families of enantioenriched molecules (e.g., heterocycles, aldehydes, and alkyl halides) with little loss of stereochemistry at both the boron-bound and 
nitrogen-bound carbons (95-99). A wide array of enamines and $\alpha$-haloboronates, which varied in amino-protecting groups and side chains, served as suitable coupling partners in this enamine reductive alkylation reaction. Reductive alkylation proceeded well with consistently good yields and high levels of enantiocontrol on the carbon adjacent to the $N$-atom with all substrates. Although mediocre diastereoselectivities on the carbon adjacent to the $B$-atom were observed in a few cases (e.g., 83-86), these two diastereoisomers could be prepared in a single reaction and then effortlessly separated through routine column chromatography.

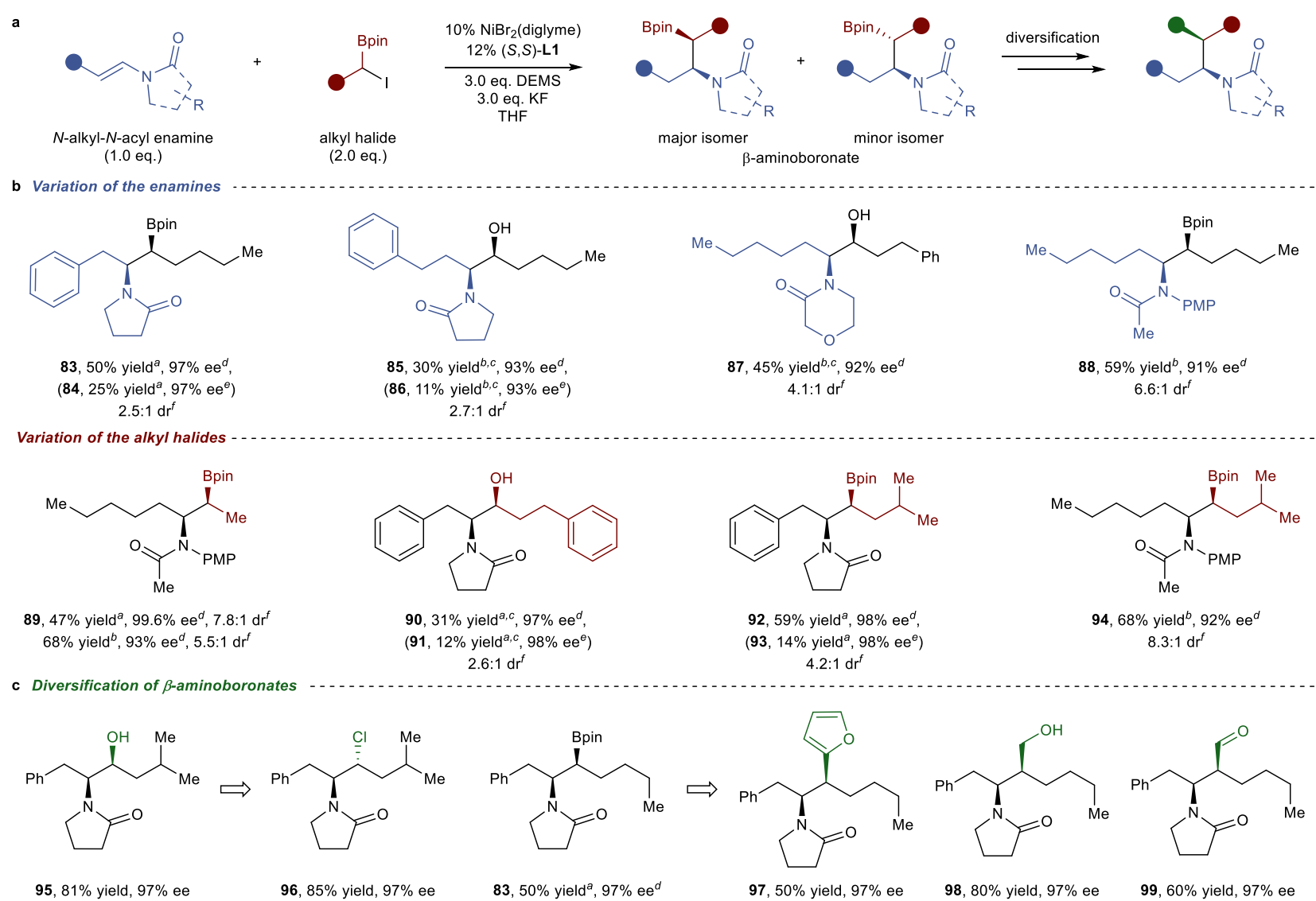

Fig. 5 Asymmetric reductive alkylation of enamines with racemic $\alpha$-haloboronates to access $\beta$-aminoboronates. a, Optimized conditions for the reductive alkylation of enamines with racemic $\alpha$-haloboronates. b, Scope of the reductive alkylation of enamines with racemic $\alpha$-haloboronates. c, Diversification of $\beta$-aminoboronates through alkylborate transformations and the Mitsunobu reaction. ${ }^{a} 25{ }^{\circ} \mathrm{C}$, 24 hours, isolated yield, $0.20 \mathrm{mmol} \mathrm{scale;}{ }^{b} 4 \mathrm{o}^{\circ} \mathrm{C}$, 24 hours, isolated yield, $0.20 \mathrm{mmol} \mathrm{scale;}{ }^{~}$ The product was isolated after the oxidization of the corresponding alkylborate; ${ }^{d}$ enantiomeric excess of major isomer after oxidization; ${ }^{e}$ enantiomeric excess of minor isomer after oxidization; f The $\mathrm{dr}$ value was determined via ${ }^{1} \mathrm{H}$ NMR analysis of crude $\beta$-aminoboronates or HPLC analysis of crude $\beta$-aminoalcohols. Bpin = boronic acid pinacol ester.

Given that chiral amine motifs exist in many small-molecule pharmaceutical agents and physiologically active natural compounds, the convenient synthesis of such molecules and medicinally valuable derivatives would convincingly demonstrate the availability and practicability of this process. A representative chiral amine 10o, the key intermediate of a new 
immunosuppressive agent, features two sterically and electronically similar alkyl substrates. The conventional method to access 100 requires time-consuming and tedious synthesis using a stoichiometric chiral allylation reagent, a poor atom-economic amino activating group, and high hydrogen pressure ${ }^{35}$. By capitalizing on the flexible retrosynthetic analysis of enamine reductive alkylation, amine 100 could be conveniently accessed through the use of readily available raw materials via an asymmetric catalytic method. This enamine reductive alkylation was also useful for the total synthesis of alkaloids, such as coniine and coniceine. The commercially available raw material 105 was employed in the reductive alkylation, and desired products 106 and 107 were obtained in an efficient fashion. Subsequent intramolecular cyclization on the accommodated alkyl chloride groups yielded the pivotal intermediates and synthesis targets (108-109). A foremost advantage of the current catalytic asymmetric enamine reductive alkylation is its substantial capacity to quickly and efficiently access 1-arylpropan-2amines (e.g., amphetamine), which are key components of central nervous system drugs. The vast array of benzyl bromides and benzyl alcohols could be easily programmed into arylpropanamines with high structural and functional diversity. Various isotope- or fluorinelabelled pharmaceutical agents, such as trifluoromethyl labelled-Carmoterol (an asthma medicine), fluorine labelled-Lisdexamfetamine (an attention deficit and hyperactivity disorder medicine), and deuterium isotope-labelled Tamsulosin (a urinary system medicine), were produced via brief synthetic routes $(\mathbf{1 1 3}, 115,118)$, highlighting the suitability of enamine reductive alkylation in drug discovery. Additionally, reductive alkylation of $N$-alkyl- $N$-acyl enamines also shows promise in tailor-made applications in the synthesis of the orexin receptor antagonist 122, which contains a chiral carbon adjacent to a tertiary amide. 


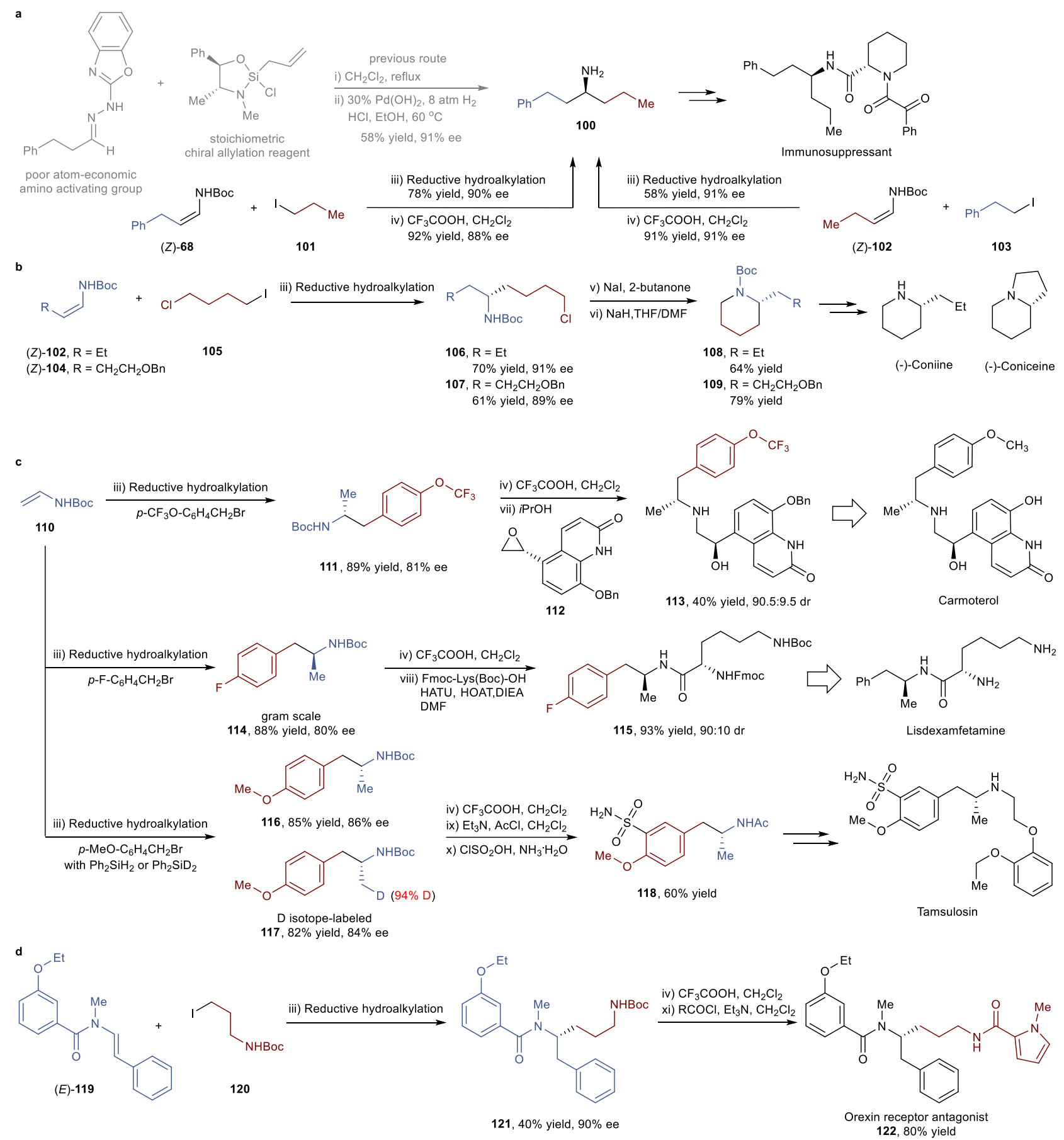

Fig. 6 Synthetic transformations of enamine reductive alkylation products. a, Comparison of enamine reductive alkylation with conventional methods for the synthesis of the key intermediate of an immunosuppressant agent. b, Synthesis of alkaloids with enamine reductive alkylation as a key step. c, Modular synthesis of isotope- or fluorine-labelled central nervous system drugs via enamine reductive alkylation. d, Formal synthesis of drug analogues that contain a chiral carbon adjusted to a tertiary amide. All yields refer to isolated yield of purified product. For more details for reaction conditions, see supplement information.

In summary, we have described a streamlined method to access chiral aliphatic amines and their derivatives through nickel-catalysed asymmetric reductive alkylation of enamines with alkyl halides. This reaction greatly complements the substrate scope of traditional chiral amine synthesis strategies, overcomes the limitation of essential and finite auxiliary groups, and expands the horizon of retrosynthetic analysis in amine synthesis. We believe that the ready 
accessibility of raw materials, convenient implementation, and efficiency of this highly stereoselective amine synthesis will lead to many applications in organic chemistry and pharmaceutical chemistry.

\section{Acknowledgements}

The authors acknowledge the support from the National Natural Science Foundation of China (21732006, 21702200, 51821006, 21927814, and 51961135104), the National Key R\&D Program of China (2017YFA0303502), and the Strategic Priority Research Program of CAS (XDB20oooooo).

\section{Author contributions}

J.-W.W. and Y.L. contributed equally to this work. X.L. and Y.F. directed the project and wrote the manuscript. Y.F. directed the DFT calculations. X.L. designed the experiments. J.-W.W. performed the experiments for the reductive alkylation of $N$-alkyl- $N$-acyl enamines. Y.L. performed the experiments for the reductive alkylation of $N-\mathrm{H}-N$-acyl enamines. W.N. performed the DFT calculations. Z.C., Z.-A.Y. and Y.-F.Z. helped to complete the experiments. All the authors participated in the preparation of the manuscript.

\section{Competing interests}

The authors declare no competing financial interests.

\section{References}

Nugent, T. C. \& El-Shazly, M. Chiral Amine Synthesis - Recent Developments and Trends for Enamide Reduction, Reductive Amination, and Imine Reduction. Adv. Synth. Catal. 352, 753-819, doi:10.1002/adsc.200900719 (2010).

2 Trowbridge, A., Walton, S. M. \& Gaunt, M. J. New Strategies for the Transition-Metal Catalyzed Synthesis of Aliphatic Amines. Chem. Rev. 120, 2613-2692, doi:10.1021/acs.chemrev.9boo462 (2020).

3 The list of Top 200 Brand Name Drugs by Retail Sales in 2019 was compiled and produced by the Njardarson group which is freely accessible to anyone as PDF files at https://njardarson.lab.arizona.edu/content/top-pharmaceuticals-poster.

4 Xie, J. H., Zhu, S. F. \& Zhou, Q. L. Transition Metal-Catalyzed Enantioselective Hydrogenation of Enamines and Imines. Chem. Rev. 111, 1713 (2011).

5 Xie, J.-H., Zhu, S.-F. \& Zhou, Q.-L. Recent advances in transition metal-catalyzed enantioselective hydrogenation of unprotected enamines. Chem. Soc. Rev. 41, 4126-4139, doi:10.1039/c2cs35007f (2012). Yasukawa, T., Masuda, R. \& Kobayashi, S. Development of heterogeneous catalyst systems for the continuous synthesis of chiral amines via asymmetric hydrogenation. Nat. Catal. 2, 1088-1092, doi:10.1038/s41929-019-0371-y (2019). 
amination. J. Am. Chem. Soc. 128, 84-86, doi:10.1021/jao57222n (2006).

Davies, H. M. L. \& Manning, J. R. Catalytic C-H functionalization by metal carbenoid and nitrenoid insertion. Nature 451, 417-424, doi:10.1038/natureo6485 (2008).

Nakafuku, K. M. et al. Enantioselective radical C-H amination for the synthesis of beta-amino alcohols. Nat. Chem. 12, 697-704, doi:10.1038/s41557-020-0482-8 (2020).

Park, Y., Kim, Y. \& Chang, S. Transition Metal-Catalyzed C-H Amination: Scope, Mechanism, and Applications. Chem. Rev. 117, 9247-9301, doi:10.1021/acs.chemrev.6boo644 (2017).

Yang, Y., Shi, S. L., Niu, D., Liu, P. \& Buchwald, S. L. Catalytic Asymmetric Hydroamination of Unactivated Internal Olefins to Aliphatic Amines. Science 349, 62-66 (2015).

Shi, S.-L., Wong, Z. L. \& Buchwald, S. L. Copper-catalysed enantioselective stereodivergent synthesis of amino alcohols. Nature 532, 353-356, doi:10.1038/nature17191 (2016).

Zhu, S., Niljianskul, N. \& Buchwald, S. L. A direct approach to amines with remote stereocentres by enantioselective $\mathrm{CuH}$-catalysed reductive relay hydroamination. Nat. Chem. 8, 144-150, doi:10.1038/nchem.2418 (2016).

Xi, Y. M., Butcher, T. W., Zhang, J. \& Hartwig, J. F. Regioselective, Asymmetric Formal Hydroamination of Unactivated Internal Alkenes. Angew. Chem. Int. Ed. 55, 776-78o, doi:10.1002/anie.201509235 (2016).

Mao, R., Frey, A., Balon, J. \& Hu, X. Decarboxylative C(sp(3))-N cross-coupling via synergetic photoredox and copper catalysis. Nat. Catal. 1, 120-126, doi:10.1038/s41929-017-0023-Z (2018). Kumar, R., Floden, N. J., Whitehurst, W. G. \& Gaunt, M. J. A general carbonyl alkylative amination for tertiary amine synthesis. Nature 581, 415-420, doi:10.1038/s41586-020-2213-o (2020). Trowbridge, A., Reich, D. \& Gaunt, M. J. Multicomponent synthesis of tertiary alkylamines by photocatalytic olefin-hydroaminoalkylation. Nature 561, 522-527, doi:10.1038/s41586-018-0537-9 (2018). Musacchio, A. J. et al. Catalytic intermolecular hydroaminations of unactivated olefins with secondary alkyl amines. Science 355, 727-730, doi:10.1126/science.aal3010 (2017).

Lindqvist, M. et al. Chiral Molecular Tweezers: Synthesis and Reactivity in Asymmetric Hydrogenation. J. Am. Chem. Soc. 137, 4038-4041, doi:10.1021/ja512658m (2015).

Li, M.-L., Yu, J.-H., Li, Y.-H., Zhu, S.-F. \& Zhou, Q.-L. Highly enantioselective carbene insertion into N-H bonds of aliphatic amines. Science 366, 990-994, doi:10.1126/science.aaw9939 (2019).

Liu, G. C., Cogan, D. A. \& Ellman, J. A. Catalytic asymmetric synthesis of tert-butanesulfinamide. Application to the asymmetric synthesis of amines. J. Am. Chem. Soc. 119, 9913-9914, doi:10.1021/ja972012Z (1997).

Fujihara, H., Nagai, K. \& Tomioka, K. Copper-amidophosphine catalyst in asymmetric addition of organozinc to imines. J. Am. Chem. Soc. 122, 12055-12056, doi:10.1021/jaoo5629g (2000).

Boezio, A. A. \& Charette, A. B. Catalytic enantioselective addition of dialkylzinc to Ndiphenylphosphinoylimines. A practical synthesis of alpha-chiral amines. J. Am. Chem. Soc. 125, 1692-1693, doi:10.1021/ja027673x (2003).

4 Lu, X. et al. Practical carbon-carbon bond formation from olefins through nickel-catalyzed reductive olefin hydrocarbonation. Nat. Commun. 7, 11129, doi:10.1038/ncomms11129 (2016).

He, S.-J. et al. Nickel-Catalyzed Enantioconvergent Reductive Hydroalkylation of Olefins with alphaHeteroatom Phosphorus or Sulfur Alkyl Electrophiles. J. Am. Chem. Soc. 142, 214-221, doi:10.1021/jacs.9bo9415 (2020).

Lu, X. et al. Nickel-Catalyzed Defluorinative Reductive Cross-Coupling of gem-Difluoroalkenes with Unactivated Secondary and Tertiary Alkyl Halides. J. Am. Chem. Soc. 139, 12632-12637, doi:10.1021/jacs.7bo6469 (2017).

Wang, X.-X., Lu, X., He, S.-J. \& Fu, Y. Nickel-catalyzed three-component olefin reductive dicarbofunctionalization to access alkylborates. Chem. Sci. 11, 7950-7956, doi:10.1039/DoSCo2054K (2020). $\mathrm{Lu}, \mathrm{X}$. et al. Nickel-catalyzed allylic defluorinative alkylation of trifluoromethyl alkenes with reductive decarboxylation of redox-active esters. Chem. Sci. 1o, 809-814, doi:10.1039/C8SC04335C (2019).

Wang, X.-X., Lu, X., Li, Y., Wang, J.-W. \& Fu, Y. Recent Advances in Nickel-Catalyzed Reductive 
Hydroalkylation and Hydroarylation of Electronically Unbiased Alkenes. Sci. China Chem., doi:10.1007/s11426-020-9838-x (2020).

30 Wang, Z., Yin, H. \& Fu, G. C. Catalytic enantioconvergent coupling of secondary and tertiary electrophiles with olefins. Nature 563, 379-383, doi:10.1038/s41586-018-o669-y (2018).

31 Xiao, J., He, Y., Ye, F. \& Zhu, S. Remote sp(3) C-H Amination of Alkenes with Nitroarenes. Chem 4, 16451657, doi:10.1016/j.chempr.2018.04.008 (2018).

32 Bera, S., Mao, R., Hu, X. Enantioselective C(sp3)-C(sp3) Cross-Coupling of Non-activated Alkyl Electrophiles via Nickel Hydride Catalysis. ChemRxiv. Preprint., doi:10.26434/chemrxiv.12040398.v1 (2020).

33 Niljianskul, N., Zhu, S. \& Buchwald, S. L. Enantioselective Synthesis of alpha-Aminosilanes by CopperCatalyzed Hydroamination of Vinylsilanes. Angew. Chem. Int. Ed. 54, 1638-1641, doi:10.1002/anie.201410326 (2015).

34 Wang, J., Liu, X. \& Feng, X. Asymmetric strecker reactions. Chem. Rev. 111, 6947-6983, doi:10.1021/cr200057t (2011).

35 Feske, M. I., Santanilla, A. B. \& Leighton, J. L. Asymmetric Allylation, Crotylation, and Cinnamylation of N-Heteroaryl Hydrazones. Org. Lett. 12, 688-691, doi:10.1021/ol9026864 (2010). 